\title{
Proyecto de transformación del calor residual de gases de combustión de un automóvil en energía eléctrica, como contribución al cuidado del medio ambiente
}

Project to transform the waste heat from the combustion gases of a car into electricity, as a contribution to the care of the environment.

Otto Fernando Balseca Sampedro. ${ }^{1}$, Diego Fernando Mayorga Pérez. ${ }^{2}$, Edwin Fernando Viteri Núñez. ${ }^{3}$

\section{Abstract. https://doi.org/10.33262/cienciadigital.v3i3.1.676}

The present investigation consists in designing a device that allows to generate a continuous voltage ( 5 volts), using a device made up of Peltier Cells, dissipaters and a base block, the suitable union of these elements will be able to charge a portable battery, which can be implemented to charge electronic devices that work with a voltage. The objective of this study is based on the Seebek Effect, which consists in the production of electric current from the contact between two different metals, two semiconductors, or a metal and a semiconductor, which are in the same circuit, due to the difference of temperature between them. Based on the above, a device was developed that can be adapted to the exhaust pipe of a car which will allow us to absorb the heat wasted by it and thus heat one side of the Peltier cell, while the other side A dissipator will be connected to the face, which will dissipate the heat through the movement of the vehicle by means of convection, thus generating a temperature difference between the two faces of the cell.

To confirm the viability of the device, an experimental analysis was carried out in which a fraction of the device was elaborated by means of a Peltier Cell, a dissipator and an aluminum block, simulating the most critical conditions that could be generated in real conditions of use with which a voltage of $2.5 \mathrm{~V}$ was obtained with a temperature difference of $70.4^{\circ} \mathrm{C}$ as a maximum value by subjecting the cell to its maximum capacity, however, we consider that we would work with a temperature

\footnotetext{
${ }^{1}$ Escuela Superior Politécnica de Chimborazo, Chimborazo, Ecuador, otto.balseca@espoch.edu.ec

${ }^{2}$ Escuela Superior Politécnica de Chimborazo, Chimborazo, Ecuador, diego.mayorga@espoch.edu.ec

${ }^{3}$ Escuela Superior Politécnica de Chimborazo, Chimborazo, Ecuador, eviteri@espoch.edu.ec
} 
difference of $54^{\circ} \mathrm{C}$, since this gives us a $2.4 \mathrm{~V}$ voltage which is not a significant variation in voltage, but it represents a safer temperature level for the durability of the cell.

Keywords: Peltier Cell, Seebek Effect, Temperature, Voltage, Dissipator.

\section{Resumen.}

La presente investigación consiste en diseñar un dispositivo que permite generar un voltaje continuo (5 voltios), utilizando un dispositivo conformado por celdas Peltier, disipadores y un bloque base, la unión adecuada de estos elementos podrán cargara una batería portátil, el cual podrá ser implementado para cargar dispositivos electrónicos que funcionen con un voltaje. El objetivo de este estudio se basa en el efecto Seebek que consiste en la producción de corriente eléctrica a partir del contacto entre dos metales diferentes, dos semiconductores, o un metal y un semiconductor, que se hallan en un mismo circuito, debido a la diferencia de temperatura entre ellos. Con base en lo expuesto anteriormente, se elaboró un dispositivo que se podrá adaptar al tubo de escape de un automóvil el cual nos permitirá absorber el calor desperdiciado por este y de esta manera calentar una de las caras de la Celda Peltier, mientras que el otro lado de la cara se conectará un disipador, este a través del movimiento del vehículo disipará el calor por medio de la convección generando así una diferencia de temperatura entre las dos caras de la celda. Para confirmar la viabilidad del dispositivo se realizó un análisis experimental en el cual se elaboró una fracción del dispositivo por medio de una Celda Peltier, un disipador y un bloque de aluminio, simulando las condiciones más críticas que se podría generar en condiciones reales de uso con los cuales se obtuvo un voltaje de $2.5 \mathrm{~V}$ con una diferencia de temperatura de $70.4{ }^{\circ} \mathrm{C}$ como un valor máximo sometiendo a la celda a su máxima capacidad, sin embargo consideramos que se trabajaría con una diferencia de temperatura de $54{ }^{\circ} \mathrm{C}$ ya que con ello se obtiene un voltaje de $2.4 \mathrm{~V}$ lo cual no es una variación significativa en voltaje, pero si representa un nivel de temperatura más seguro para la durabilidad de la celda.

Palabras claves: Celda Peltier, Efecto Seebek, Temperatura, Voltaje, Disipador.

\section{Introducción.}

La generación de energía para cargar un celular mediante el aprovechamiento del calor desperdiciado por el tubo de escape de los motores de combustión interna contribuye a la conservación del medio ambiente, dado que a nivel mundial los vehículos han aumentado radicalmente. (Cevallos E, 2015). En Ecuador, según la norma técnica para emisiones a la atmósfera de fuentes fijas, un automóvil no debe emitir más de 100 mg/Nm3 de Dióxido de 
Carbono lo que conlleva a buscar maneras de aprovechar la energía del motor de combustión interna. (ANT, 2013). Las grandes industrias automovilísticas han centrado su interés en el aprovechamiento de esta energía como es el caso de BMW la cual está desarrollando este Sistema con un aproximado de 200 voltios para un mejor rendimiento de automóviles (Fernández C., 2016).

Además, varios investigadores han logrado obtener energía eléctrica mediante la variación de temperatura en el sistema de escape de los vehículos tal es el caso del equipo de investigadores del Instituto Tecnológico de Massachusetts (MIT por sus siglas en inglés) ha desarrollado un generador termoeléctrico capaz de producir electricidad limpia y respetuosa con el medio ambiente gracias a las variaciones de temperatura. (Jiménez, 2018).

Sin embargo, los dispositivos termoeléctricos, que pueden generar energía eléctrica cuando hay una diferencia de temperatura entre uno de sus lados y el otro, han sido objeto de muchas investigaciones durante los últimos años. Es así como, un equipo de trabajo ha ideado una forma novedosa de convertir las fluctuaciones de temperatura en energía eléctrica. En vez de necesitar dos temperaturas diferentes al mismo tiempo, el nuevo sistema aprovecha las oscilaciones de la temperatura ambiental que suceden durante dentro del tubo de escape de los vehículos. (NCYT, 2018).

Uno de los casos más particulares de generación de energía eléctrica mediante la variación de temperatura es un resonador térmico. Según recoge MIT News en System draws power from daily temperature swings "un resonador térmico es algo que puede dejarse sobre una mesa y producir electricidad a partir de lo que parece ser nada. Estamos rodeados por oscilaciones de temperatura de todas las frecuencias, todo el tiempo. Son una fuente de energía sin explotar." (Digital, E, 2018).

Se ha analizado que en el sistema de escape que consta de colector, catalizador, silenciador tiene una perdida térmica de un 32 por ciento de energía (Plata Gerardo, Badillo Carlos, Olivares Miguel, 2014) es por ello que surge la necesidad de diseñar un dispositivo que nos permita cargar el celular dentro del automóvil, para lo cual se utilizaran las celdas TE (termoeléctrica) las que son utilizadas en los sistemas de refrigeración .Dichas celdas funcionan con el efecto Peltier, siendo su contrario el Efecto Seebek el cual funciona con una variación de temperatura entre el tubo de escape y un sistema de refrigeración. La temperatura para nuestro propósito esta entre los 100 a $700{ }^{\circ} \mathrm{C}$. (Fernández C., 2016)

Las empresas con cogeneración presentan mayor grado de resistencia a la crisis gracias al uso eficiente de los recursos y la mejor medioambiental, claves para la competitividad y la capacidad exportadora. Los datos del año 2011 confirman que las industrias con cogeneración vieron crecer su producción eléctrica un $2 \%$ lo que consta una mayor actividad en sus industrias asociadas frente a una caída del -1,4\% en el índice de producción industrial 
manufacturera y el $-1,6 \%$ en la industria de suministro de energía eléctrica, gas y otros. Según estimaciones de ACOGEN, la producción alcanzó los 33.700 GWh y se exportaron 24.865 GWh con una potencia instalada de 6.114 MW. (Rodríguez, R., 2012)

Actualmente, la electricidad se obtiene principalmente de la quema de combustibles fósiles. Están surgiendo proyectos innovadores que relacionan termodinámica con electricidad, es decir, proyectos que plantean recuperar calor para convertirlo en energía eléctrica. Así se ofrecen alternativas menos contaminantes, aunque, por el momento, de bajo rendimiento. (Jiménez, S., 2016)

\section{Marco Teórico}

\section{A. Dispositivo para la generación de energía.}

Los dispositivos TE (Termoeléctricos), pueden transformar el calor directamente en energía eléctrica y también puede actuar como enfriadores de estado sólido. Los avances en la tecnología de TE puede tener un impacto significativo en la industria del automóvil en términos de ahorro de combustible, mejoras mediante la generación de electricidad a partir del calor residual y de aire acondicionado de alta eficiencia. En primer lugar, la tecnología de TE tiene la capacidad de utilizar las decenas de kilovatios de las pérdidas de calor en los vehículos para generar electricidad sin carga del motor añadido, esto ayudará a producir energía eléctrica suficiente para energizar un dispositivo que funcione con el voltaje de salida.

\section{B. Temperatura del sistema de tubo de escape.}

Un motor de combustión interna no es capaz de transformar toda la energía en movimiento existen ciertas perdidas, las cuales se disipan en forma de calor hacia la atmosfera. Se detallan las partes del tubo de escape y sus temperaturas con el fin de determinar la energía calorífica que poseen dichos componentes, el cual nos servirá para realizar una variación de temperatura y por ende variación de Voltaje mediante el Principio de Seebek. (Joan,2014).

\section{Partes del sistema de escape.}

Colector de Escape Es la primera parte por donde el gas sale del motor, luego de haber realizado la combustión, la temperatura se encuentra en un rango $202.7^{\circ} \mathrm{C}$ a $178.7^{\circ} \mathrm{C}$, lo que es energía térmica desperdiciada, luego de este parte del sistema del tubo de escape pasa catalizador. 
Catalizador Esta parte es la responsable de transformar gases contaminantes de la combustión a gases menos nocivos para el ambiente, esta parte del sistema de escape se encuentra a una temperatura de $154.6{ }^{\circ} \mathrm{C}$ a $118.6{ }^{\circ} \mathrm{C}$. Luego de pasar esta parte se tiene el tubo de escape posterior.

TUBO DE ESCAPE POSTERIOR Cuenta con un silenciador el cual reduce los sonidos, producidos por la combustión, cabe recalcar que esta parte menor al rendimiento al motor, puesto que limita la salida de gases del motor, esto se encuentra a una temperatura de $94.53^{\circ} \mathrm{C}$ a $58.47^{\circ} \mathrm{C}$.

\section{Ecuaciones.}

\section{EFECTO DE SEEBEK}

Se refiere a la emisión de electricidad en un circuito eléctrico compuesto por conductores diferentes, a diferentes temperaturas. Dicho principio se halla representado por la fórmula:

$$
\alpha=\frac{V}{\Delta T}
$$

Donde:

$\mathrm{V}=$ Voltaje termodinámico [Voltios]

$\alpha=$ Coeficiente de Seebeck $[\mu(\mathrm{V} / \mathrm{K})]$

$\Delta \mathrm{T}=$ Temperatura del gradiente $[\mathrm{K}]$

Este el principio que se utilizará para generar energía mediante la variación de temperatura existente en el tubo de escape y el sistema de refrigeración. (Echeverría, M., 2015)

RAZON DE TRANSFERENCIA DE CALOR

$$
\begin{gathered}
Q_{\text {total aleta }}=Q_{\text {libre de aleta }}+Q_{\text {aleta }} \\
Q=h A_{\text {libre de aleta }}\left(T_{s}-T_{b}\right)+h \eta_{\text {aleta }} A_{\text {aleta }}\left(T_{s}-T_{b}\right)
\end{gathered}
$$

Donde:

$\mathrm{Q}=$ Razón de transferencia de calor. [W]

$\mathrm{h}=$ Coeficiente convectivo. $\left[\mathrm{W} / \mathrm{m}^{2} \mathrm{~K}\right]$

$\mathrm{A}=$ Área. $\left[\mathrm{m}^{2}\right]$

Ts $=$ Temperatura de la superficie. $[\mathrm{K}]$

$\mathrm{T} \infty=$ Temperatura ambiente. $[\mathrm{K}]$

$\eta=$ Eficiencia de la aleta.

TEMPERATURA DE PELÍCULA 
Es la temperatura promedio que existe en la temperatura de la superficie y la temperatura ambiente.

$$
T_{p}=\frac{T_{s}+T_{\infty}}{2}
$$

\section{REYNOLDS}

$$
R e=\frac{V L}{v}
$$

Donde:

$\mathrm{V}=$ velocidad del fluido $[\mathrm{m} / \mathrm{s}]$

$\mathrm{L}=$ longitud del disipador $[\mathrm{m}]$

$v=V$ iscosidad cinemática en $\left[\mathrm{m}^{2} / \mathrm{s}\right]$

\section{NUSSELT}

$$
N u=\frac{h L}{k}
$$

Donde:

$\mathrm{h}=$ Coeficiente convectivo. $\left[\mathrm{W} / \mathrm{m}^{2} \mathrm{~K}\right]$

$\mathrm{L}=$ Longitud de la celda [m]

$\mathrm{k}=$ conductividad térmica en [W/ $\mathrm{m} \mathrm{K}$ ]

\section{RESISTENCIA TÉRMICA}

$$
Q=\frac{T_{s}-T_{\infty}}{R_{\text {real }}}
$$

Donde:

$\mathrm{Q}=$ Razón de transferencia de calor [W]

Ts $=$ Temperatura de la superficie $[\mathrm{K}]$

T $\infty=$ Temperatura ambiente $[\mathrm{K}]$

Rreal $=$ Resistencia Térmica real $[\mathrm{K} / \mathrm{W}]$

\section{E. Datos calculados.}

Tabla 1. Propiedades del aire a presión de 1 atm [6] 


\begin{tabular}{|c|c|c|c|c|c|c|}
\hline $\begin{array}{l}\text { Temp. } \\
\mathrm{T}\left[{ }^{\circ} \mathrm{C}\right]\end{array}$ & $\begin{array}{c}\text { Calor } \\
\text { Específico, } \\
\text { Cp [J/kgK] }\end{array}$ & $\begin{array}{l}\text { Conductividad } \\
\text { térmica } k \\
{[\mathrm{~W} / \mathrm{m} \mathrm{K}]}\end{array}$ & $\begin{array}{c}\text { Difusividad } \\
\text { térmica } \\
\text { [m2/s2] }\end{array}$ & $\begin{array}{l}\text { Viscosidad } \\
\text { dinámica } \\
{[\mathrm{kg} / \mathrm{m} \mathrm{s}]}\end{array}$ & $\begin{array}{c}\text { Viscosidad } \\
\text { cinemática } \\
\text { [m2/s] }\end{array}$ & $\begin{array}{l}\text { Número de } \\
\text { Prandtl }\end{array}$ \\
\hline 40 & 1007 & 0,02662 & 2,35E-05 & 1,92E-05 & $1,70 E-05$ & $7,26 \mathrm{E}-01$ \\
\hline 45 & 1007 & 0,02699 & $2,42 \mathrm{E}-05$ & $1,94 \mathrm{E}-05$ & $1,75 \mathrm{E}-05$ & $7,24 \mathrm{E}-01$ \\
\hline
\end{tabular}

Con estos datos se procede a realizar la interpolación para encontrar los valores requeridos para la realización de los cálculos correspondientes. Estos resultados se muestran en la tabla 2.

Tabla 2. Interpolación

\begin{tabular}{cccc}
\hline $\begin{array}{c}\text { Temp. } \\
\text { T, }\left[{ }^{\circ} \mathbf{C}\right]\end{array}$ & $\begin{array}{c}\text { Conductividad } \\
\text { térmica } \mathbf{k} \\
{[\mathbf{W} / \mathbf{m} \text { K] }}\end{array}$ & $\begin{array}{c}\text { Viscosidad } \\
\text { cinemática } \\
{[\mathbf{m} 2 / \mathbf{s}]}\end{array}$ & $\begin{array}{c}\text { Número de } \\
\text { Prandtl } \\
\mathbf{P r}\end{array}$ \\
\hline 40 & 0,002662 & $1,702 \mathrm{E}-05$ & 0,7255 \\
41,2844 & 0,02671 & $1,714 \mathrm{E}-05$ & 0,7251 \\
45 & 0,02699 & $1,750 \mathrm{E}-05$ & 0,7241 \\
\hline
\end{tabular}

\section{F. Figuras y tablas.}

Tabla 3. Correlación empírica para el Número de Nusselt promedio, para convección forzada sobre cilindros circulares y no circulares en flujo cruzado [43]

\begin{tabular}{cccc}
\hline Sección transversal del fluido & Fluido & $\begin{array}{l}\text { Rango de } \\
\text { Reynolds }\end{array}$ & $\begin{array}{c}\text { Número de } \\
\text { Nusselt }\end{array}$ \\
\hline Placa vertical & Gas & $4000-15000$ & $0,228\left(\operatorname{Re}^{\wedge} 0,731\right)\left(\operatorname{Pr}^{\wedge} 1 / 3\right)$ \\
\hline
\end{tabular}

Las figuras muestran las dimensiones del disipador necesario para el cumplimiento de los objetivos propuestos en este trabajo (Fig. 1) y las dimensiones del disipador que se utilizara para la experimentación el cual se aproxima más a las dimensiones del disipador obtenido en los cálculos (Fig. 2).

Fig. 1. Disipador de calor para placas de circuito impreso. [8] 


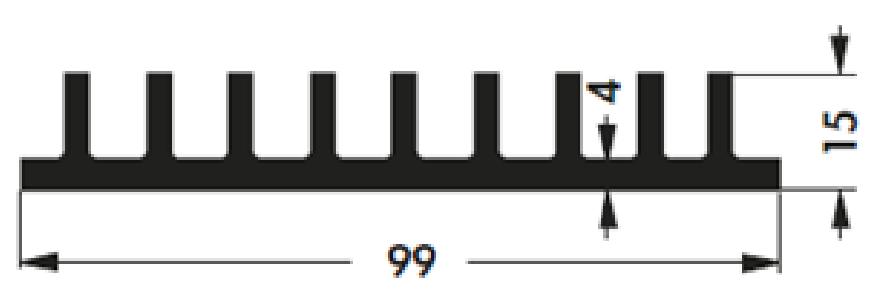

Fig. 2. Dimensiones de disipador obtenido en el mercado de aluminio.

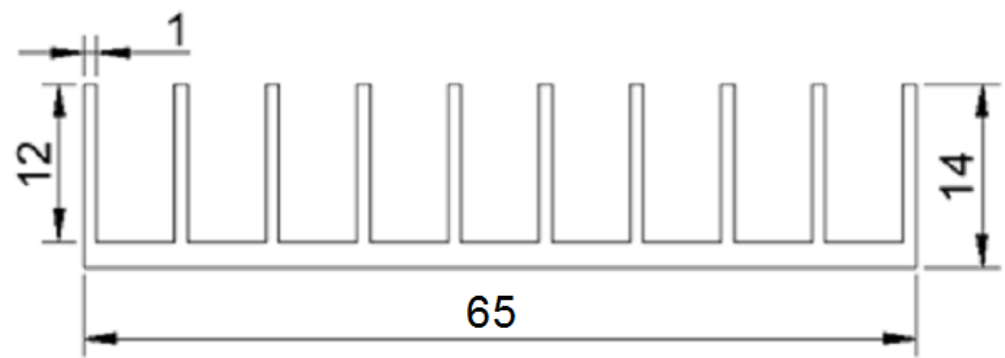

Para determinar la confiabilidad del disipador encontrado en el mercado se realizó una comparación de resistencia térmica del disipador obtenido en el mercado con el del catálogo obteniendo una resistividad semejante por lo cual se determinó que este si es viable para el dispositivo.

Fig. 3. Gráfica Resistencia térmica versus longitud del disipador. [8]

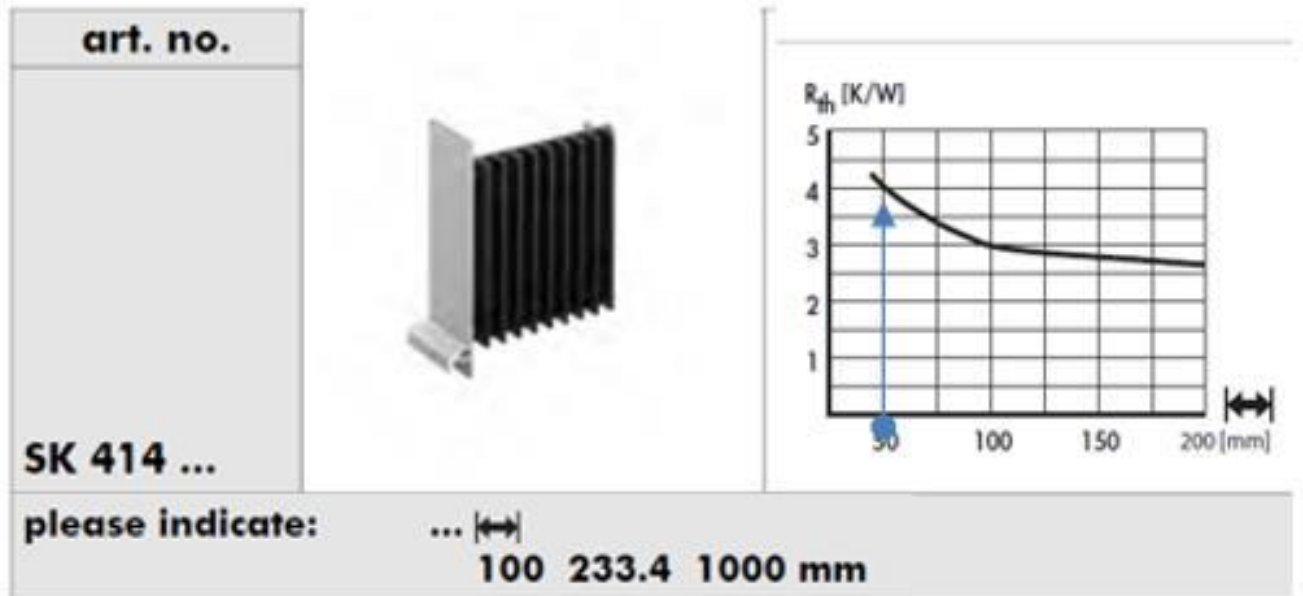

En la figura 4 se muestra la gráfica para determinar la resistencia térmica forzada ya que el tipo de convección que tenemos presente en el medio de trabajo del dispositivo es forzado debido al flujo del aire frio en la parte del disipador. 
Resistencia térmica de cualquier perfil con convección forzada.

$$
R_{t h K f}=a R_{t h K}
$$

Donde:

RthKf $=$ Resistencia térmica con convección forzada

RthK = Resistencia térmica con convección natural

$\mathrm{a}=$ Factor de proporción

Fig. 4. Resistencia térmica de cualquier perfil con convección forzada. [8]

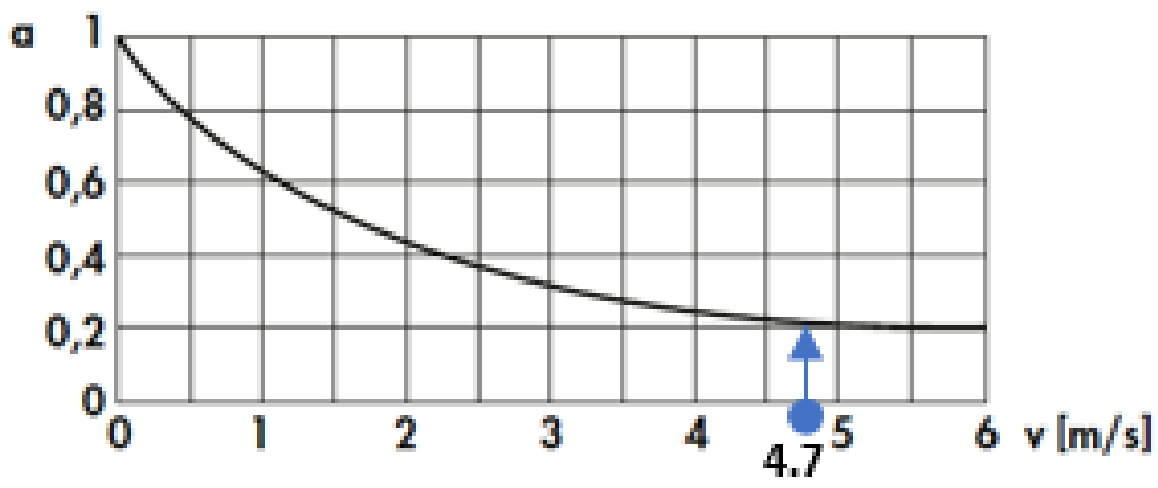

Tabla 4. Resultados para la obtención Coeficiente Seebeck.

\begin{tabular}{cc}
\hline Voltaje (V) & Delta T $\left({ }^{\circ} \mathrm{C}\right)$ \\
\hline 2,4 & 54 \\
2,41 & 56 \\
2,42 & 57,2 \\
2,43 & 59,2 \\
2,44 & 61,3 \\
2,45 & 63,4 \\
2,46 & 64,6 \\
2,47 & 64,8 \\
2,48 & 66,4 \\
2,49 & 72,4 \\
2,5 & 70,4 \\
\hline
\end{tabular}


Utilizando la ecuación 1 para encontrar el coeficiente de Seebeck y empleando los datos expuestos en la tabla 4, obtenidos experimentalmente se obtiene un coeficiente de Seebeck de:

$$
\alpha=0.039 \frac{\mathrm{V}}{{ }^{\circ} \mathrm{C}}
$$

\section{ANALISIS EXPERIMENTAL}

\section{A. Materiales.}

- Elementos de medida:

1. Multímetro. (x2)

2. Pirómetro.

3. Anemómetro.

- Elementos de prueba:

1. Pila Peltier

2. Disipador

3. Chapa metálica

4. Cables de conexiones

5. Bloque de aluminio

6. Fuente de calor controlada

7. Pasta térmica

8. Cinta adhesiva.

9. Diodo 1N4007S (x2)

10. Ventilador de $5 \mathrm{~V}$

11. Fuente de $5 \mathrm{~V}$

12. Soporte para instrumentos

13. Cables de conexión

14. Resistencia de $325 \Omega$

\section{B. Procedimiento.}

Cuando se inserta un material del tipo físico, químico o biológico en elementos hídricos estos afectan a la calidad del agua, a continuación, se muestran varios factores que inciden en esta:

1. Se procede a armar el dispositivo eléctrico.

2. Se coloca el dispositivo sobre la fuente de calor, el ventilador en dirección que el aire impacte directo al disipador.

3. Se conecta los diodos en antiparalelo como medida de protección, el multímetro en serie, este medirá la intensidad de corriente, consecutivamente la resistencia de 325 $\Omega$ y se procede a cerrar el circuito con el negativo de la Pila Peltier.

4. El otro multímetro será conectado en paralelo para medir la intensidad. 
5. El sensor de temperatura será colocado dentro del dispositivo en el disipador (Cara fría del dispositivo).

6. El pirómetro apuntara a la cara del bloque de aluminio (cara caliente del dispositivo)

7. Para una mejor apreciación de datos se procederá a filmar las pantallas de cada uno de los dispositivos.

\section{Resultados}

En la tabla 5 se muestran los resultados del mínimo teórico para determinar la selección del disipador que permita cumplir con los requerimientos necesario que ayuden a obtener un diferencial de temperatura necesario para alcanzar un voltaje aceptable en el dispositivo.

Tabla 5. Resultados para selección del disipador.

\begin{tabular}{cccc}
\hline Variables & Real & Teórico & Unidades \\
\hline $\begin{array}{c}\text { Velocidad } \\
\text { Velocidad de } \\
\text { temperatura }\end{array}$ & 4,7 & 4,7 & $\mathrm{~m} / \mathrm{s}$ \\
$\begin{array}{c}\text { Temperatura } \\
\text { del lado frio }\end{array}$ & 62,7 & 62,7 & ${ }^{\circ} \mathrm{C}$ \\
$\begin{array}{c}\text { Temperatura de } \\
\text { la pelicula }\end{array}$ & 41,28844 & 17,3 & ${ }^{\circ} \mathrm{C}$ \\
$\begin{array}{c}\text { Reynolds } \\
\text { Nusselt }\end{array}$ & 13710,618 & 13710,618 & - \\
$\begin{array}{c}\text { Coefiente } \\
\text { convectivo }\end{array}$ & 216,566 & 216,566 & - \\
$\begin{array}{c}\text { Área de la aleta } \\
\text { Área libre de la } \\
\text { aleta }\end{array}$ & 0,0108 & 115,69 & $\mathrm{C}$ \\
$\begin{array}{c}\text { Eficiencia de la } \\
\text { aleta }\end{array}$ & $2,76 \times 10-3$ & 0,0099 & $\mathrm{~W} /\left(\mathrm{m}^{\wedge} 2 \mathrm{~K}\right)$ \\
$\begin{array}{c}\text { Razón de la } \\
\text { transferencia de } \\
\text { calor }\end{array}$ & 28,183 & $3,15 \times 10-3$ & $\mathrm{~m} 2$ \\
$\begin{array}{c}\text { Resistencia } \\
\text { térmica }\end{array}$ & 1,52 & 0,4707 & $\mathrm{~K} / \mathrm{W}$ \\
\hline
\end{tabular}


En la tabla 6 se presenta una muestra de los valores más relevantes obtenidos en el proceso de la experimentación, por medio del cual se realizaron las medidas del voltaje y la corriente obtenida a las diferentes $\Delta \mathrm{T}$ en una celda Peltier.

Gracias a estos resultados podemos determinar el comportamiento de la celda a diferentes cambios de temperatura y de esta manera buscar la forma de estabilizarlo en una tención de $5 \mathrm{~V}$ que es lo recomendable para la carga de un dispositivo móvil.

Tabla 6. Resultados experimentales obtenidos con el dispositivo.

\begin{tabular}{cccccc}
\hline No & $\begin{array}{c}\text { T caliente } \\
{\left[{ }^{\circ} \mathrm{C}\right]}\end{array}$ & $\begin{array}{c}\text { T fria } \\
{\left[{ }^{\circ} \mathrm{C}\right]}\end{array}$ & $\begin{array}{c}\text { Delta T } \\
{\left[{ }^{\circ} \mathbf{C}\right]}\end{array}$ & $\begin{array}{c}\text { Voltaje } \\
{\left[{ }^{\circ} \mathbf{C}\right]}\end{array}$ & $\begin{array}{c}\text { Amperaje } \\
{[\mathbf{m a}]}\end{array}$ \\
\hline 1 & 46,9 & 43,4 & 3,5 & 0,55 & 1,6 \\
2 & 48,8 & 46,7 & 2,1 & 0,6 & 1,75 \\
3 & 53,3 & 50 & 3,3 & 0,7 & 2,04 \\
4 & 57,4 & 53,4 & 4 & 0,8 & 2,34 \\
5 & 62,7 & 56,7 & 6 & 0,9 & 2,65 \\
6 & 67,5 & 56,7 & 10,8 & 1 & 2,93 \\
7 & 72,3 & 60 & 12,3 & 1,1 & 3,23 \\
8 & 76,9 & 63,4 & 13,5 & 1,2 & 3,52 \\
9 & 81,4 & 63,4 & 18 & 1,3 & 3,8 \\
10 & 86,1 & 66,7 & 19,4 & 1,4 & 4,11 \\
11 & 92,1 & 70 & 22,1 & 1,5 & 4,41 \\
12 & 97,7 & 73,4 & 24,3 & 1,6 & 4,7 \\
13 & 103,2 & 73,4 & 29,8 & 1,7 & 4,98 \\
14 & 109,4 & 80 & 29,4 & 1,8 & 5,29 \\
15 & 115,6 & 83,4 & 32,2 & 1,9 & 5,58 \\
16 & 125,3 & 90 & 35,3 & 2 & 5,88 \\
17 & 131 & 93,3 & 37,7 & 2,1 & 6,17 \\
18 & 139,2 & 96,6 & 42,6 & 2,2 & 6,47 \\
19 & 149 & 100 & 49 & 2,3 & 6,77 \\
20 & 160 & 106 & 54 & 2,4 & 7,06 \\
21 & 180,4 & 110 & 70,4 & 2,5 & 7,35 \\
22 & 207,8 & 110 & 97,8 & 2,51 & 7,37 \\
\hline & & & & &
\end{tabular}


Fig. 5. Curva polinomial de grado 6 obtenida por los puntos tomados en la experimentación.

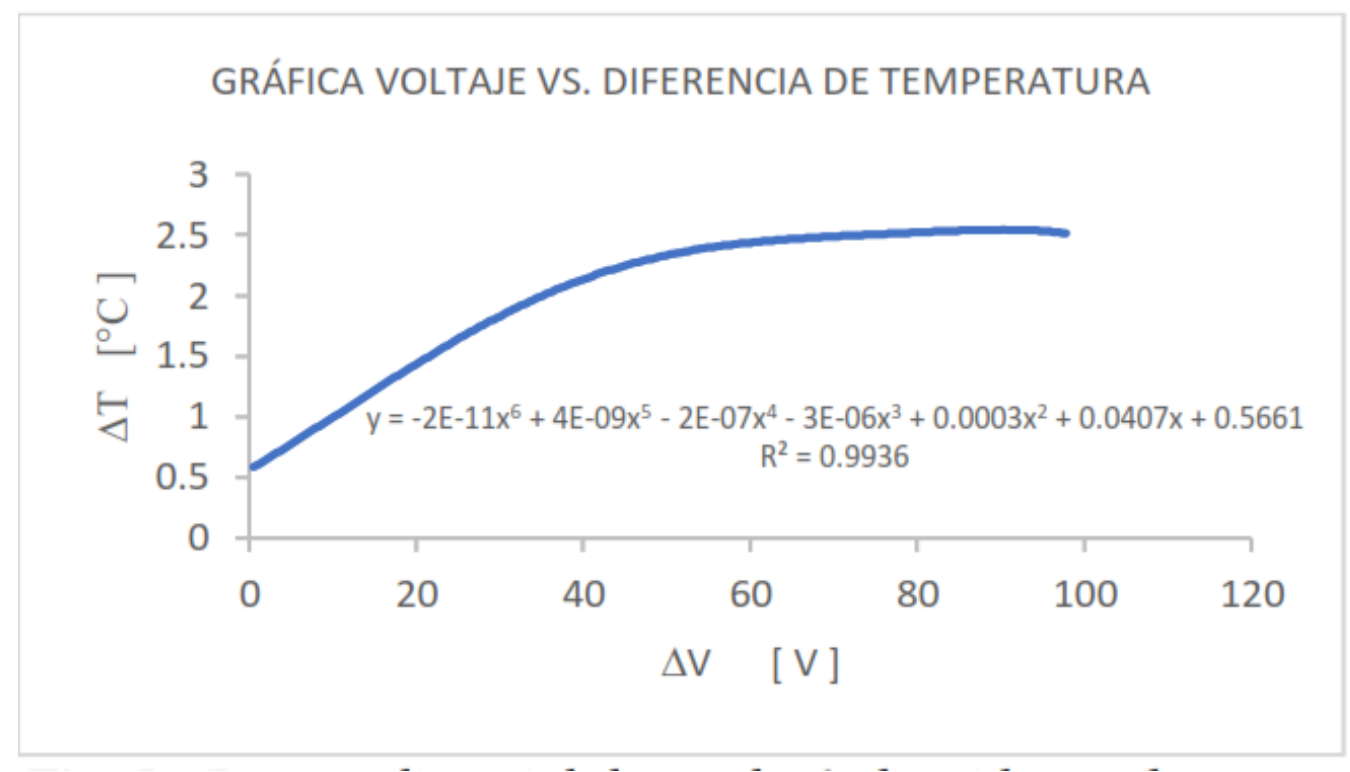

\section{Simulación.}

Se procedió a realizar una simulación en ANSYS, para comparar resultados experimentales con la simulación, para una mayor confiabilidad en los resultados obtenidos. La figura 6 muestran los resultados obtenidos en la simulación en ANSYS.

Fig. 6. Variación de la temperatura a través del dispositivo.

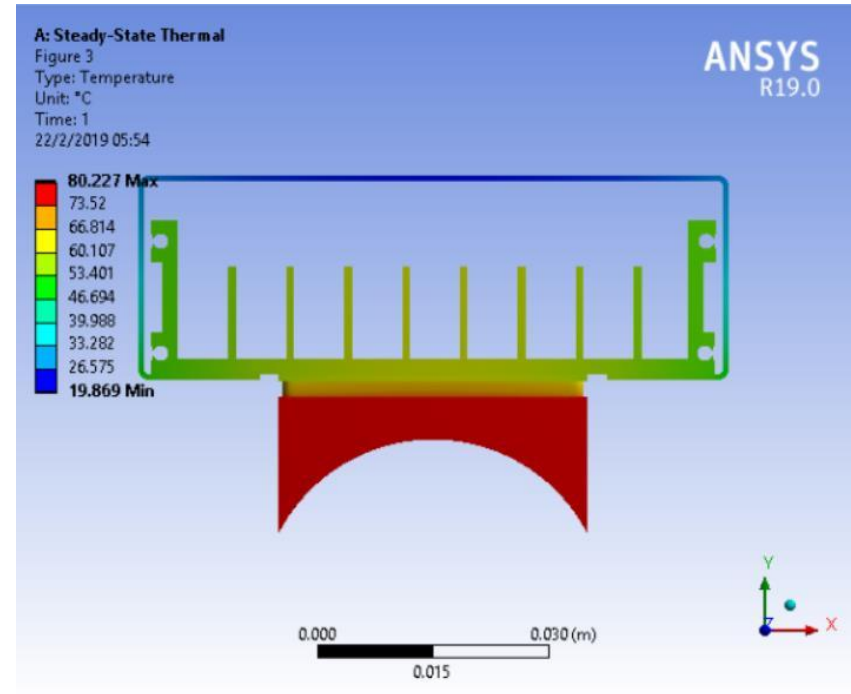


Como se puede observar en la figura 7 se puede notar el cambio de la temperatura desde la cara más fría a la más caliente de la celta Peltier, lo que indica que si se genera una diferencia de temperatura de acorde con los datos experimentales obtenidos en el análisis experimental.

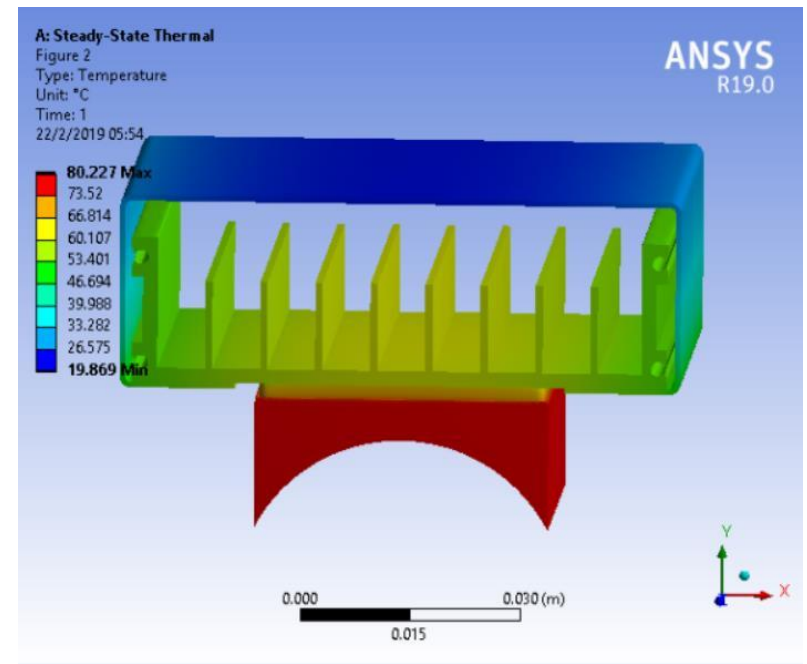

Con la ayuda del Software ANSYS se determinó la temperatura, en diferentes puntos a lo largo del eje Y, obteniendo los siguientes valores expuestos en la tabla 7, de igual manera en la figura 8 se muestra la ubicación de los puntos tomados para la realización.

Tabla 7. Valores registrados a lo largo del eje y.

\begin{tabular}{cc}
\hline $\begin{array}{c}\text { Longitud } \\
{[\mathrm{mm}]}\end{array}$ & $\begin{array}{c}\text { Temperatura } \\
{\left[{ }^{\circ} \mathrm{C}\right]}\end{array}$ \\
\hline 0 & 80 \\
1,327 & 73,028 \\
2,45 & 69,999 \\
3,573 & 66,667 \\
5,206 & 62,685 \\
6,431 & 62,65 \\
7,758 & 62,598 \\
9,289 & 62,541 \\
10,718 & 62,497 \\
11,432 & 62,478 \\
12,147 & 62,462 \\
13,371 & 62,437 \\
14,188 & 62,423 \\
15,311 & 62,409 \\
16,229 & 62,401 \\
\hline
\end{tabular}




$\begin{array}{ll}17,046 & 62,396 \\ 17,862 & 62,393\end{array}$

Fig. 10. Valores registrados en un corte paralelo al plano $\mathrm{Y}-\mathrm{Z}$ cortado en el origen.

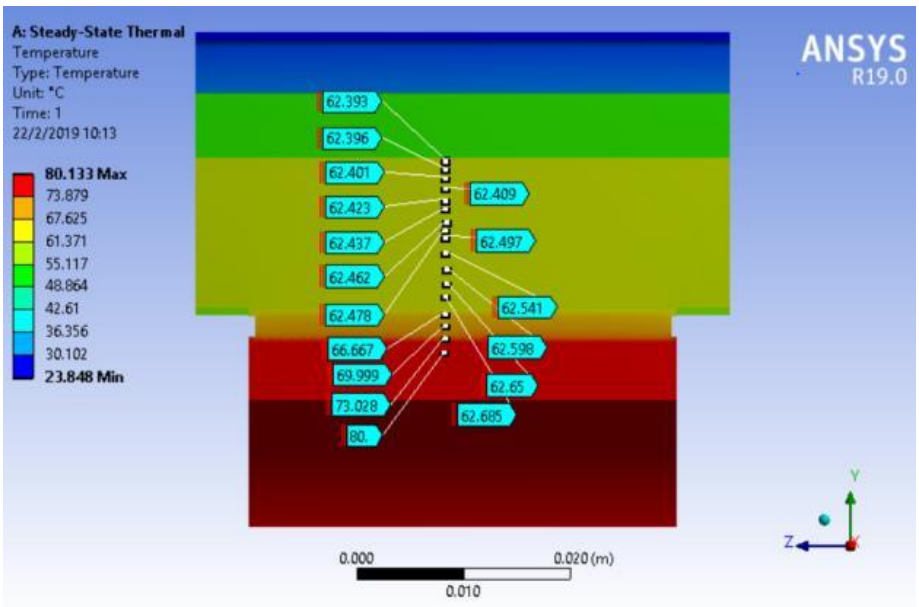

En base a los datos mostrados en la tabla 7, se procede a graficar (Fig. 11) las variaciones de temperatura en función de la distancia a lo largo del eje X, y de esta manera ver las distintas temperaturas a lo largo del eje $\mathrm{Y}$.

Fig. 11. Curva temperatura - Longitud que describe el cambio de temperatura a lo largo del eje Y.

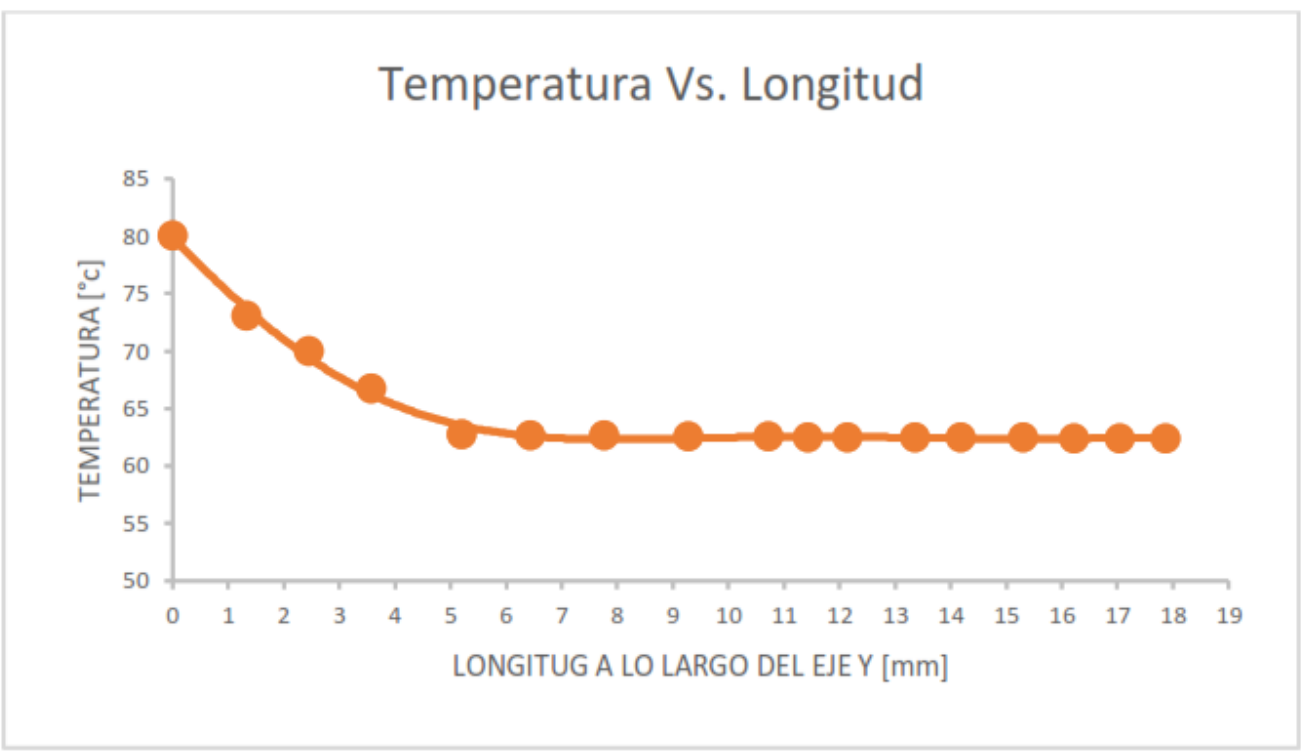




\section{Conclusiones.}

- Según los datos mostrados podemos observar que experimentalmente a una diferencia de temperatura de $70.4^{\circ} \mathrm{C}$ se puede obtener tensión de $2.5 \mathrm{~V}$ y una corriente de $7.35 \mathrm{~mA}$. la cual es semejante al valor obtenido en los datos teóricos, esto permite verificar la viabilidad del aprovechamiento de la energía residual del tubo de escape en un dispositivo para cargar celulares.

- Al realizar el experimento en un rango inferior al que estaría sometido en la realidad nos asegura el correcto funcionamiento del dispositivo ya que las pruebas fueron realizadas con una velocidad de viento de $2.5 \mathrm{~m} / \mathrm{s}$ dentro de un ambiente cerrado, esto significa que al estar a la intemperie la variación de temperatura se producirá en un menor tiempo.

- La celta Peltier TEC1-12706 en un rango de variación de temperatura [10 a 70] ${ }^{\circ} \mathrm{C}$ produce una tención máxima de $2.5 \mathrm{~V}$, si sobrepasamos este rango la tensión tiende a permanecer constante.

- Mediante la experimentación se comprobó que el disipador empleado en este dispositivo cumple con las necesidades requeridas para disipar el calor requerido que nos permite obtener la diferencia de temperatura necesaria para el funcionamiento del dispositivo.

- Por la experimentación realizada se pudo comprobar que el voltaje obtenida por una celda es de $2.5 \mathrm{~V}$, por tanto, para alcanzar nuestros objetivos debemos conectar tres dispositivos en serie a lo largo del tubo de escape.

\section{Referencias bibliográficas.}

[1] Ángeles, A., \& Juan, J. (2016). Instrumentación de un sistema de paneles solares para el diseño de un controlador de temperatura basado en el efecto Peltier-Seebeck (Doctoral dissertation).

[2] Argüelles Guerrero, Rodrigo. (2011). Análisis experimental de un generador Peltier y sus procesos irreversibles (doctoral dissertation).

[3] Arroyo. (2013). Medida de parámetros termoeléctricos en un sistemaconstituido por dispositivos Peltier-Seebeck.

[4] Beltrán Pitarch, B. (2017). Nuevo método de medida de la conductividad y difusividad térmica usando un módulo termoeléctrico.

[5] Bollati, E., \& Corporex, S. A. (2007). Generadores termoeléctricos. Petrotecnia, 1, 85.

[6] Cengel. F (1992). Transferencia de Calor. Mexico : Mc Graw Hill.

[7] Chover, G. (2013). Diseño de un sistema de control de temperatura para un prototipo de generación de frío y calor, basado en células Peltier (Doctoral dissertation). 
[8] Díaz García, S. (2017). Análisis de viabilidad de recuperación de energía mediante efecto termoeléctrico.

[9] Duque Solís, M. (2013). Diseño de paneles generadores de electricidad por efecto Seebeck (Doctoral dissertation, Universidad EIA).

[10] Echeverria, M. (2015). 41, Quito: s.n., 2015, Vol. 17.

[11] Esparza Zárate, I. (2016). Estudio y simulación de nuevas estructuras de módulos termoeléctricos para generación de energía eléctrica mediante efecto Seebeck.

[12] Fernández, J. (2014). [En línea] septiembre de 2014. [Citado el: 8 de febrero de 2019.]

upcommons.upc.edu/bitstream/handle/2099.1/23357/memoria\%20Joan\%20Fernandez\%20 PFC.pdf.

[13] Flórez, J. O. V., \& Martínez, A. F. (2017). Implementación de la Celda Peltier en fuentes térmicas de calor residuales, para aprovechamiento de generación de energía eléctrica y climatización por frio en el hogar. VISIÓN-Revista de Investigaciones UNICIENCIA, 2(2), 62-69.

[14] Fouille, M. (2006). Compendio de electrónica. España: Editorial Coizareu.

[15] García Rivera, J. D., \& Live Sevilla, J. A. (2013). Generación de Energía por Gradiente de Temperatura (Bachelor's thesis, QUITO/EPN/2013).

[16] González Martín, S. (2011). Estudio y caracterización de aleaciones Zn-Sb para aplicaciones termoeléctricas.

[17] Guamán, G., Fabricio, L., Palomino, U., \& Pablo, J. (2014). Análisis del comportamiento de módulos termoeléctricos para transformación del calor existente en el colector de escape a energía eléctrica en un motor G10 (Bachelor's thesis).

[18] Jiménez, H. (2016). Desarrollo de un sistema de conversión de calor en energía eléctrica.

[19] Jiménez Sara. 2016. Desarrollo de un sistema de conversión e calor en energía eléctrica. Madrid: s.n., 2016. La recuperación termoeléctrica del calor residual, una fuente de energía ambientalmente amigable.

[20] Joan. (2014). Análisis del aprovechamiento de calores residuales de los gases de escape del motor térmico mediante efecto Seebeck en unión $\mathrm{Cu}$-al upc. Scielo. 
[21] Juanicó, L., \& Rinalde, F. (2010). Análisis comparativo de paneles termoeléctricos y fotovoltaicos para electrificación de hogares aislados. Interciencia, 35(2).

[22] Leal, J. F., León, M. M., \& Sepúlveda, S. B. (2015). El diodo Schottky como atenuador del efecto Seebeck en una celda Peltier para un control PID de temperatura. Entre Ciencia e Ingeniería, 9(18), 75-83.

[23] Mario, E. (2015). Materiales termoeléctricos que permiten transformar calor residual de automóviles que permiten transformar calor residual de automóviles en energía eléctrica. Scielo.

[24] Medrano Parra, L. A. (2016). Diseño, construcción y evaluación de un generador termoeléctrico acoplado a una estufa ecológica (Doctoral dissertation, Instituto de Ciencias Básicas y aplicadas-Ingeniería en Energías Renovables-UNICACH).

[25] Montejo, V. M. R., Lara, M. N., \& Garfias, J. D. J. N. S. (2017). Obtención de clústeres metálicos con propiedades Superconductoras para su evaluación del efecto Seebeck. Jóvenes en la ciencia, 2(1), 1409-1412.

[26] Palacios, R., Arenas, A., Vazquez, J., Pagola, F. L., \& Pecharromán, R. R. (2006). Caracterización experimental de un Paramento Transparente Activo Termoeléctrico (PTA). Climamed 2006. Congrès Méditerranéen des Climaticiens. p. 305-314. Lyon, France, 20-21 November 2006.

[27] Patterson, G., \& Sobral, M. (2007). Efecto Peltier. Departamento de Física FCEyN, Universidad de Buenos Aires.

[28] Pérez Asiain, J. (2014). Estudio experimental de la resistencia térmica en función de la relación entre el área de módulos Peltier y el área de la base de un heat pipe.

[29] Pérez Valdeblanquez, H. E., Parra, M., \& Dubiel, Y. Generación de Condensado con Celdas de Peltier.

[30] Pérez, M., \& Alberto, J. (2018). Estudio de aplicación de células Peltier para la obtención de electricidad en automóviles (Doctoral dissertation).

[31] Plata Gerardo, Badillo Carlos, Olivares Miguel. (2014). Pistas educativas. [En línea] octubre de 2014. [Citado el: 16 de febrero de 2019.] itcelaya.edu.mx/ojs/index.php/pistas/article/download/1390/1202.

[32] Ramírez, C. R., Lizarazo, G. M., \& Duarte, E. V. (2017). Termoelectricidad: uso de las celdas Peltier en el campo de la refrigeración y sus principales aplicaciones. INVENTUM, 12(22), 9-16. 
[33] Rivera, E. M. L. (2008). Generación Eléctrica Por Pila Termoeléctrica - Efecto Seebeck. Isagogé, (5), 10.

[34] Rodríguez, J. (2010). 41, Bogotá: Momento, 2010. Materiales termoeléctricos que permiten transformar calor residual de automóviles en energía eléctrica.

[35] Rodríguez, R. (2012). Instalación para el aprovechamiento energético de los gases de escape de once motores de biogas. Madrid: s.n., 2012.

[36] Rof Ballús, A. (2015). Estudio y parametrización de un generador termoeléctrico (Bachelor's thesis, Universitat Politècnica de Catalunya).

[37] Romo, C., Medrano, C., Romero, H., Arvizu, J., Huacuz, J. \& Beltran, J. (2012). Comisión Federal de Electricidad. [En línea] Octubre de 2012. [Citado el: 16 de febrero de 2019.] ineelmx/docu/Guia-RUS.pdf.

[38] Sandoval, \& Espinosa, E. (2008). Celdas Peltier: Una alternativa para sistemas de enfriamiento con base. Instituto de Electrónica. Universidad Tecnológica de la Mixteca.

[39] Ángeles, A., \& Juan, J. (2016). Instrumentación de un sistema de paneles solares para el diseño de un controlador de temperatura basado en el efecto PeltierSeebeck (Doctoral dissertation).

[40] Santacruz López, H., López Puente, S. I., Esquivel Castro, Y., Solís Martínez, E. E., \& Ortíz Baltazar, J. A. (2017). Alternativa Peltier. Solsona, J. A. C. MATERIALES TERMOELECTRICOS. APLICACIONES PARA LA REFRIGERACION Y LA GENERACION DE ELECTRICIDAD.

[41] Yánez, M. E. E., \& Romero, E. M. C. (2018). MATERIALES TERMOELÉCTRICAS QUE PERMITEN TRANSFORMAR CALOR RESIDUAL DE AUTOMÓVILES EN ENERGÍA ELÉCTRICA. Ciencia, 17(1).

[42] Zavala Pavón, P. E. (2011). Potencial termodinámico para dispositivos termoeléctricos (Doctoral dissertation).

[43] Zukauskas, F. Jakob, G., (1942). Transferencia de Calor y Masa. Correlaciones empíricas de Nusselt. Referencia 14.

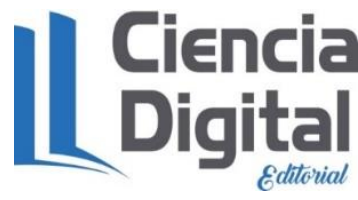




\section{PARA CITAR EL ARTÍCULO INDEXADO.}

Balseca Sampedro, O., Mayorga Pérez, D., \& Viteri Núñez, E. (2019). Proyecto de transformación del calor residual de gases de combustión de un automóvil en energía eléctrica, como contribución al cuidado del medio ambiente. Ciencia Digital, 3(3.1), 54-73. https://doi.org/10.33262/cienciadigital.v3i3.1.676

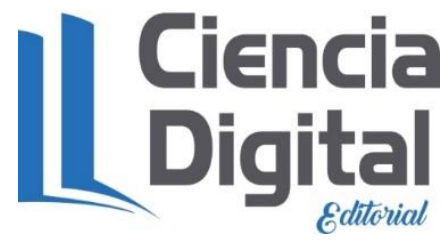

El artículo que se publica es de exclusiva responsabilidad de los autores y no necesariamente reflejan el pensamiento de la Revista Ciencia Digital.

El artículo queda en propiedad de la revista y, por tanto, su publicación parcial y/o total en otro medio tiene que ser autorizado por el director de la Revista Ciencia Digital.
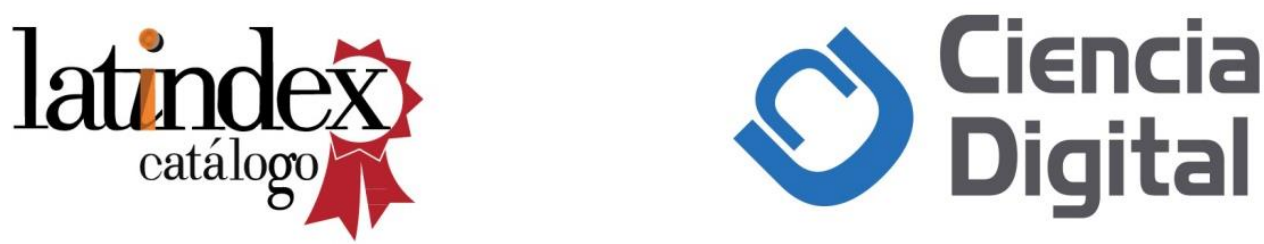